\title{
ON ERRORS OF A UNIFIED FAMILY OF APPROXIMATION FORMS OF BIVARIATE CONTINUOUS FUNCTIONS
}

\author{
CHUNG-SIUNG KAO
}

\begin{abstract}
Approximation forms for a regular bivariate functions $f(x, y)$ were obtained by putting expectation on a convergent bivariate stochastic sequence for which some proper error bounds are herein derived to evaluate the applicability of the approximation forms when actually applied to be approximates of regular bivariate functions
\end{abstract}

\section{Introduction}

As is widely understood, Bernstein polynomials has become an important tool for doing computer aided geometric design (CAGD) in industries for designing automobiles and aircrafts, etc., as well as in producing simulated motion pictures. While univariate Bernstein polynomials may be useful for designing curves, bivariate Bernstein polynomials appear to be equally useful for designing desirable surfaces. In fact, more extensive and generalized bivariate approximation forms have been shown by Kao (2002), with which further flexibility and effectiveness on their use to CAGD may be expected. In addition, it is normally feasible to obtain bivariate density estimates by applying an approximation form for regular bivariate functions. Naturally, error bounds for the bivariate approximation forms should be available in order that idea about how good the approximates will be and how fast the convergence of the approximation forms to the actual value of the approximated function can be obtained. In this work, the author make available some appropriate error bounds to meet the needs. In univariate case, recently Ghosal (2001) investigated the convergence rates when Bernstein polynomials were applied to estimating densities. Impens and Vernaeve (2001) gave some asymptotics in regard to Bernstein approximation forms when the approximated function satisfies certain Lipschitz conditions. Furthermore, Petrone (1999) applied Bernstein polynomials to density estimation under a Bayesian nonparametric framework. Regarding convergence of Bernstein approximation form, Farouki (1999) investigated its convergent inversion approximations. All such published pieces of intensive work on Bernstein polynomials regarding their error and convergence offer strong indication about the important need for use of Bernstein polynomials in CAGD. Attempts were recently made to expand the

Received June 25, 2002; revised October 7, 2002.

2000 Mathematics Subject Classification. 60E05, 41A15.

Key words and phrases. Bivariate Bernstein polynomials, approximation forms, expectation, Law of large numbers, error bounds. 
consideration into multivariate situation, primarily regarding how the multivariate version should be formulated and their asymptotic expansions were provided. Notable work include the results by Abel and Ivan (2000) specifically on simplex domain, and by Walz (2000). Since bivariate treatment normally leads the way for any general multivariate treatment, formulation for multivariate approximation forms and corresponding results on error bounds of the approximation forms are expected to be obtainable without too much difficulty through this bivariate work. Also, it is important to note that bivariate Bernstein polynomials appear to be only one in a family of approximation forms proposed by this author. There are other probably more powerful approximation forms in this family that can be very useful to computer aided geometric design.

\section{A Family of Approximation Forms}

Assume $f$ to be a continuous function, and according to practicality in applications $f$ is also assumed to be uniformly bounded. Then let there be a sequence of identically and independently distributed (iid) random variables $X_{1}, X_{2}, \ldots, X_{m}$ with mean $x$ and finite variance $\sigma^{2}>0$, and another sequence of iid random variables $Y_{1}, Y_{2}, \ldots, Y_{n}$ with mean $y$ and finite variance $w^{2}>0$. Without loss of generality, we assume $x>0$ and $y>0$. Then let $S_{m}^{(X)}$ and $S_{n}^{(Y)}$ denote the sums of $X_{i}$ 's and $Y_{j}$ 's respectively, i.e.

$$
S_{m}^{(x)}=X_{1}+X_{2}+\cdots+X_{m} \quad \text { and } \quad S_{n}^{(Y)}=Y_{1}+Y_{2}+\cdots+Y_{n}
$$

According to the strong law of large numbers in probability, we have

$$
\begin{aligned}
& \frac{S_{m}^{(X)}}{m} \longrightarrow x \text { as } m \rightarrow \infty \text { almost surely } \\
\text { and } \quad & \frac{S_{n}^{(Y)}}{n} \longrightarrow y \text { as } n \rightarrow \infty \text { almost surely }
\end{aligned}
$$

For any bivariate function $f$ which is continuous at $(x, y)$, it then follows that

$$
f\left(\frac{S_{m}^{(X)}}{m}, \frac{S_{n}^{(Y)}}{n}\right) \rightarrow f(x, y) \text { as }(m, n) \rightarrow(\infty, \infty) \text { almost surely }
$$

Since $f(x, y)$ is assumed to be uniformly bounded for any $(x, y)$, the dominated convergence theorem implies that

$$
f(x, y)=\lim _{(m, n) \rightarrow(\infty, \infty)} E f\left(\frac{S_{m}^{(X)}}{m}, \frac{S_{n}^{(Y)}}{n}\right)
$$

if $f$ is continuous at $(x, y)$.

It should be noted that with same argument, we have

$$
\left.f(x, y)=\lim _{n \rightarrow \infty} E f\left(\frac{S_{n}^{(X)}}{n}, \frac{S_{n}^{(Y)}}{n}\right)\right)
$$


However, we primarily apply Formula (2.1) to obtain a family of interesting and useful approximation forms as previously given by Kao (2002). The approximation forms so obtained include the following:

\section{Approximation Form I}

By setting $X_{i}$ 's to be iid extended Bernoulli trials, i.e. $P\left\{X_{i}=t\right\}=p$ and $P\left\{X_{i}=\right.$ $0\}=1-p$, and $Y_{j}$ 's to be iid extended Bernoulli trials with $P\left\{Y_{j}=s\right\}=q$ and $P\left\{Y_{j}=0\right\}=1-q$, where $t>0, s>0,0 \leq p, q \leq 1$, we can derive that

$$
\begin{aligned}
f(x, y)= & \lim _{(m, n) \rightarrow(\infty, \infty)} \sum_{\substack{0 \leq i \leq m \\
0 \leq j \leq n}} f\left(\frac{i t}{m}, \frac{j s}{n}\right) . \\
& \left(\begin{array}{c}
m \\
i
\end{array}\right)\left(\begin{array}{c}
n \\
j
\end{array}\right)\left(\frac{x}{t}\right)^{i}\left(1-\frac{x}{t}\right)^{m-i}\left(\frac{y}{s}\right)^{j}\left(1-\frac{y}{s}\right)^{n-j}
\end{aligned}
$$

where $t$ and $s$ are arbitrary such that $0 \leq x<t$ and $0 \leq y<s$. This form can be regarded as bivariate Bernstein form.

\section{Approximation Form II}

By setting $X_{i} / t, 1 \leq i \leq m$ to be iid negative binomial random variables with distribution $N B(K, p)$ and $Y_{j} / s, 1 \leq j \leq n$, to be iid negative binomial random variables with distribution $N B(M, q)$ where $t>0$ and $s>0,0 \leq p, q \leq 1, K$ and $M$ are positive integers. Then $X_{i}$ 's are independent of $Y_{j}$ 's, $E\left(X_{i}\right)=x=\frac{t \bar{K}}{p}$ and $E\left(Y_{j}\right)=y=\frac{s M}{q}$. If follows that

$$
\begin{aligned}
f(x, y) & =\lim _{(m, n) \rightarrow(\infty, \infty)} \operatorname{Ef}\left(\frac{1}{m} \sum_{i=1}^{m} X_{i}, \frac{1}{n} \sum_{j=1}^{n} Y_{j}\right) \\
& =\lim _{(m, n) \rightarrow(\infty, \infty)} \sum_{\substack{m K \leq i<\infty \\
n M \leq j<\infty}} f\left(\frac{i t}{m}, \frac{j s}{n}\right)\left(\begin{array}{c}
i-1 \\
m K-1
\end{array}\right) . \\
& \left(\begin{array}{c}
j-1 \\
n M-1
\end{array}\right)\left(\frac{t K}{x}\right)^{m K}\left(1-\frac{t K}{x}\right)^{i-m K}\left(\frac{s M}{y}\right)^{n M}\left(1-\frac{s M}{y}\right)^{j-n M}
\end{aligned}
$$

for any $(x, y)$ such that $0<t<\frac{x}{K}$ and $0<s<\frac{y}{M}$, with $t$ and $s$ both being arbitrary.

This form can be regarded as bivariate negative binomial form.

\section{Approximation Form III}

By setting $X_{i}$ 's to be iid Poisson $(x)$ and $Y_{j}$ 's to be iid Poisson( $(y)$ with the $X_{i}$ 's being independent of the $Y_{j}$ 's, where $x>0$ and $y>0$, then we can derive to obtain that

$$
f(x, y)=\lim _{(m, n) \rightarrow(\infty, \infty)} \sum_{0 \leq i, j<\infty} f\left(\frac{i}{m}, \frac{j}{n}\right) \frac{(m x)^{i}(n y)^{j}}{i ! j !} e^{-(m x+n y)}
$$


for any $(x, y)$ in first quadrant of $\mathbf{R}^{2}$.

This form can be regarded as bivariate Poisson form.

\section{Approximation Form IV}

By setting $X_{i}$ 's to be iid normally distributed $N\left(x, \sigma^{2}\right)\left(\sigma^{2}>0\right)$ and $Y_{j}$ 's to be iid normally distributed $N\left(y, w^{2}\right)\left(w^{2}>0\right)$ and the $X_{i}$ 's being independent of the $Y_{j}$ 's, then we can obtain that

$$
f(x, y)=\lim _{(m, n) \rightarrow(\infty, \infty)} \frac{\sqrt{m n}}{\sigma w} \int_{-\infty}^{\infty} \int_{-\infty}^{\infty} f(t, s) \phi\left(\frac{\sqrt{m}(t-x)}{\sigma}\right) \phi\left(\frac{\sqrt{n}(s-y)}{w}\right) d t d s
$$

where $\phi$ is the probability density function of standard normal distribution. This form can be regarded as bivariate normal form.

\section{Approximation Form V}

By setting $X_{i}$ 's to be iid exponential random variables with parameter $\frac{1}{x}>0$ and $Y_{j}$ 's to be iid exponential random variables with parameter $\frac{1}{y}>0$, and that the $X_{i}$ 's are independent of the $Y_{j}$ 's, then we can obtain that

$$
\begin{aligned}
f(x, y)= & \lim _{(m, n) \rightarrow(\infty, \infty)} \frac{x^{-m} y^{-n}}{(m-1) !(n-1) !} \int_{0}^{\infty} \int_{0}^{\infty} f\left(\frac{t}{m}, \frac{s}{n}\right) . \\
& t^{m-1} s^{n-1} e^{-\left(\frac{t}{x}+\frac{s}{y}\right)} d t d s \text { for any }(x, y) .
\end{aligned}
$$

This form can be regarded as bivariate gamma form.

All of the bivariate approximation forms obtained in above may be applicable at user's discretion. Each of them may be suitable specifically for certain type of available discrete data values of $f$ to reach an approximation of $f(x, y)$.

\section{Error Bounds for the Bivariate Approximation Forms}

For purpose of simplicity in the arguments to follow we assume that function $f$ has continuous and bounded second derivatives in either argument for any point in the defined domain. Then due to the fact that $f\left(\frac{S_{m}^{(X)}}{m}, \frac{S_{n}^{(Y)}}{n}\right)$ converges to $f(x, y)$ almost surely, where $x=E\left(X_{i}\right), y=E\left(Y_{j}\right), S_{m}^{(X)}=X_{1}+X_{2}+\cdots+X_{m}$ and $S_{n}^{(Y)}=Y_{1}+Y_{2}+\cdots+Y_{n}$, we may have

$$
f\left(\frac{S_{m}^{(X)}}{m}, \frac{S_{n}^{(Y)}}{n}\right)=f(x, y)+\nabla f(x, y) \underset{\sim}{d^{\prime}}+\frac{1}{2} \underset{\sim}{d} H(\theta, \xi) \underset{\sim}{d^{\prime}}
$$

where $\underset{\sim}{d}$ is the row vector $\left(\frac{S_{m}^{(X)}}{m}-x, \frac{S_{n}^{(Y)}}{n}-y\right), \nabla f(x, y)$ denotes the gradient of $f$ at $(x, y)$ and $H(\theta, \xi)$ denotes the Hermitian of $f$ at $(\theta, \xi)$ with $\theta$ in between $\frac{S_{m}^{(X)}}{m}$ and $x$, and $\xi$ in between $\frac{S_{n}^{(Y)}}{n}$ and $y$. In addition, $(\theta, \xi)$ approaches $(x, y)$ as $(m, n) \rightarrow(\infty, \infty)$. 
Therefore, by taking expectation we have

$$
\begin{aligned}
E f\left(\frac{S_{m}^{(X)}}{m}, \frac{S_{n}^{(Y)}}{n}\right) & =f(x, y)+\nabla f(x, y) E\left(\underset{\sim}{d^{\prime}}\right)+\frac{1}{2} E\left[\underset{\sim}{d} H(\theta, \xi) \underset{\sim}{d^{\prime}}\right] \\
& =f(x, y)+\frac{1}{2} E\left[\underset{\sim}{d} H(\theta, \xi) \underset{\sim}{\left.d^{\prime}\right]}\right.
\end{aligned}
$$

since $E(\underset{\sim}{d})=(0,0)^{\prime}$.

We assume that the second derivatives of $f$ are uniformly bounded by $C$, i.e. $\frac{\partial^{2}}{\partial x^{2}} f$, $\frac{\partial^{2}}{\partial y^{2}} f$ and $\frac{\partial^{2}}{\partial x \partial y} f$ are all bounded by $C$ uniformly. Then it follows that

$$
\begin{aligned}
& E\left[\underset{\sim}{d} H(\theta, \xi) \underset{\sim}{d^{\prime}}\right] \\
& \leq C\left[E\left(\frac{S_{m}^{(X)}}{m}-x\right)^{2}+2 E\left|\left(\frac{S_{m}^{(X)}}{m}-x\right)\left(\frac{S_{n}^{(Y)}}{n}-y\right)\right|+E\left(\frac{S_{n}^{(Y)}}{n}-y\right)^{2}\right] \\
& \leq C\left\{\operatorname{Var}\left(\frac{S_{m}^{(X)}}{m}\right)+2\left[\operatorname{Var}\left(\frac{S_{m}^{(X)}}{m}\right) \operatorname{Var}\left(\frac{S_{n}^{(Y)}}{n}\right)\right]^{\frac{1}{2}}+\operatorname{Var}\left(\frac{S_{n}^{(Y)}}{n}\right)\right\} \\
& =C\left[\left(\operatorname{Var}\left(\frac{S_{m}^{(X)}}{m}\right)\right)^{\frac{1}{2}}+\left(\operatorname{Var}\left(\frac{S_{n}^{(Y)}}{n}\right)\right)^{\frac{1}{2}}\right]^{2}
\end{aligned}
$$

where the second inequality holds according to Schwarz inequality regarding to expectation of product of two random variables.

It is important to note that when $n$ is sufficiently large, we have $(\theta, \xi) \approx(x, y)$ since $\left(\frac{S_{m}^{(X)}}{m}, \frac{S_{n}^{(Y)}}{n}\right)$ approaches $(x, y)$, and therefore

$$
f(x, y) \approx A_{m, n}(x, y)-\frac{1}{2} E\left[\underset{\sim}{d} H(x, y) \underset{\sim}{d^{\prime}}\right]
$$

where $A_{m, n}(x, y)=E f\left(\frac{S_{m}^{(X)}}{m}, \frac{S_{n}^{(Y)}}{n}\right), \underset{\sim}{d}=\left(\frac{S_{m}^{(X)}}{m}-x, \frac{S_{n}^{(Y)}}{n}-y\right)$ and $H(x, y)$ denotes the Hermitian of $f$ at $(x, y)$.

By applying (3.1) and (3.2) to each of the bivariate approximation forms provided in the previous section, we may immediately obtain error bound for each of the bivariate approximation forms as in what follows.

\section{Bivariate Bernstein Form:}

It is apparent that

$$
\operatorname{Var}\left(X_{i}\right)=t^{2} p(1-p)=x(t-x) \quad \text { and } \quad \operatorname{Var}\left(Y_{j}\right)=y(s-y)
$$

which implies that

$$
\operatorname{Var}\left(\frac{S_{m}^{(X)}}{m}\right)=\frac{1}{m} t^{2} p(1-p)=\frac{x(t-x)}{m} \quad \text { and } \quad \operatorname{Var}\left(\frac{S_{n}^{(Y)}}{n}\right)=\frac{y(s-y)}{n}
$$


Therefore, by (3.1) and (3.2) we have

$$
\begin{aligned}
& \left|f(x, y)-\sum_{\substack{0 \leq i \leq m \\
0 \leq j \leq n}} f\left(\frac{i t}{m}, \frac{j s}{n}\right)\left(\begin{array}{c}
m \\
i
\end{array}\right)\left(\begin{array}{c}
n \\
j
\end{array}\right)\left(\frac{x}{t}\right)^{i}\left(1-\frac{x}{t}\right)^{m-i}\left(\frac{y}{s}\right)^{j}\left(1-\frac{y}{s}\right)^{n-j}\right| \\
\leq & \frac{C}{2}\left[\frac{x(t-x)}{m}+\frac{y(s-y)}{n}+2 \sqrt{\frac{x y(t-x)(s-y)}{m n}}\right]
\end{aligned}
$$

for any $(x, y)$, where $(t, s)$ is arbitrary such that $0 \leq x<t$ and $0 \leq y<s$.

\section{Bivariate Negative Binomial Form:}

It is apparent that

$$
\operatorname{Var}\left(X_{i}\right)=\frac{t^{2} K(1-p)}{p^{2}}=\frac{x(x-t K)}{K} \quad \text { and } \quad \operatorname{Var}\left(Y_{j}\right)=\frac{y(y-s M)}{M}
$$

which implies that

$$
\operatorname{Var}\left(\frac{S_{m}^{(X)}}{m}\right)=\frac{t^{2} K(1-p)}{m p^{2}}=\frac{x(x-t K)}{m K} \quad \text { and } \quad \operatorname{Var}\left(\frac{S_{n}^{(Y)}}{n}\right)=\frac{y(y-s M)}{n M}
$$

Therefore, by (3.1) and (3.2) we have

$$
\begin{aligned}
& \mid f(x, y)-\sum_{\substack{m K \leq i<\infty \\
n M \leq j<\infty}} f\left(\frac{i t}{m}, \frac{j s}{n}\right)\left(\begin{array}{c}
i-1 \\
m K-1
\end{array}\right)\left(\begin{array}{c}
j-1 \\
n M-1
\end{array}\right)\left(\frac{t K}{x}\right)^{m K}\left(1-\frac{t K}{x}\right)^{i-m K} . \\
& \quad\left(\frac{s M}{y}\right)^{n M}\left(1-\frac{s M}{y}\right)^{j-n M} \mid \\
& \leq \frac{C}{2}\left[\frac{x(x-t K)}{m K}+\frac{y(y-s M)}{n M}+2 \sqrt{\frac{x y(x-t K)(y-s M)}{m n K M}}\right]
\end{aligned}
$$

for any $(x, y)$, where $(t, s)$ is arbitrary such that $0<t<\frac{x}{K}$ and $0<s<\frac{y}{M}$.

\section{Bivariate Poisson Form:}

It is apparent that

$$
\operatorname{Var}\left(X_{i}\right)=x \quad \text { and } \quad \operatorname{Var}\left(Y_{j}\right)=y \quad(x>0, y>0)
$$

which implies that

$$
\operatorname{Var}\left(\frac{S_{m}^{(X)}}{m}\right)=\frac{x}{m} \text { and } \operatorname{Var}\left(\frac{S_{n}^{(Y)}}{n}\right)=\frac{y}{n}
$$


Therefore, by (3.1) and (3.2) we have

$$
\begin{aligned}
& \left|f(x, y)-\sum_{0 \leq i, j<\infty} f\left(\frac{i}{m}, \frac{j}{n}\right) \frac{(m x)^{i}(n y)^{j}}{i ! j !} e^{-(m x+n y)}\right| \\
& \leq \frac{C}{2}\left(\frac{x}{m}+\frac{y}{n}+2 \sqrt{\frac{x y}{m n}}\right)
\end{aligned}
$$

for any $(x, y)$ such that $x>0$ and $y>0$.

\section{Bivariate Normal Form:}

It is apparent that

$$
\operatorname{Var}\left(X_{i}\right)=\sigma^{2} \quad \text { and } \operatorname{Var}\left(Y_{j}\right)=w^{2} \quad\left(\sigma^{2}>0, w^{2}>0\right)
$$

which implies that

$$
\operatorname{Var}\left(\frac{S_{m}^{(X)}}{m}\right)=\frac{\sigma^{2}}{m} \quad \text { and } \quad \operatorname{Var}\left(\frac{S_{n}^{(Y)}}{n}\right)=\frac{w^{2}}{n}
$$

Therefore, by (3.1) and (3.2) we have

$$
\begin{aligned}
& \left|f(x, y)-\frac{\sqrt{n m}}{\sigma w} \int_{-\infty}^{\infty} \int_{-\infty}^{\infty} f(t, s) \phi\left(\frac{\sqrt{m}(t-x)}{\sigma}\right) \phi\left(\frac{\sqrt{n}(s-y)}{w}\right) d t d s\right| \\
\leq & \frac{C}{2}\left(\frac{\sigma^{2}}{m}+\frac{w^{2}}{n}+2 \frac{\sigma w}{\sqrt{m n}}\right)
\end{aligned}
$$

for any $(x, y)$ in $\mathbf{R}^{2}$, where $\phi$ is the probability density function of standard normal distribution.

\section{Bivariate Gamma Form:}

It is apparent that $\operatorname{Var}\left(X_{i}\right)=x^{2}$ and $\operatorname{Var}\left(Y_{j}\right)=y^{2}(x>0, y>0)$, which implies that

$$
\operatorname{Var}\left(\frac{S_{m}^{(x)}}{m}\right)=\frac{x^{2}}{m} \quad \text { and } \operatorname{Var}\left(\frac{S_{n}^{(Y)}}{n}\right)=\frac{y^{2}}{n}
$$

Therefore, by (3.1) and (3.2) we have

$$
\begin{aligned}
& \left|f(x, y)-\frac{x^{-m} y^{-n}}{(m-1) !(n-1) !} \int_{0}^{\infty} \int_{0}^{\infty} f\left(\frac{t}{m}, \frac{s}{n}\right) t^{m-1} s^{n-1} e^{-\left(\frac{t}{x}+\frac{s}{y}\right)} d t d s\right| \\
\leq & \frac{C}{2}\left(\frac{x^{2}}{m}+\frac{y^{2}}{n}+\frac{2 x y}{\sqrt{m n}}\right)
\end{aligned}
$$

for any $x>0$ and $y>0$. 
As pointed out before at Formula (3.3), when $m$ and $n$ are sufficiently large we have the approximation forms $A_{m, n}(x, y)$ differ from $f(x, y)$ by $\left.\underset{2}{2} \underset{\sim}{d} H(x, y) \underset{\sim}{d^{\prime}}\right]$ asymptotically, which is of the order of magnitude $O\left(\max \left(\frac{1}{m}, \frac{1}{n}\right)\right)$. In addition to the almost equality of Formula (3.3), its derivation also give light to the following theorems.

Theorem 1. If $f(x, y)$ is a linear form of $x$ and $y$ individually, then for any bivariate approximation form $A_{m, n}(x, y)$ given herein we have

$$
A_{m, n}(x, y)=f(x, y)
$$

for any $(x, y)$ in the defined domain of $f(x, y)$.

Proof. Since $E\left(X_{i}\right)=x, E\left(Y_{j}\right)=y$ and $S_{m}^{(X)}=\sum_{i=1}^{m} X_{i}, S_{n}^{(Y)}=\sum_{j=1}^{n} Y_{j}$, we see that $E\left(\frac{S_{m}^{(X)}}{m}\right)=x$ and $E\left(\frac{S_{n}^{(Y)}}{n}\right)=y$. When $f(x, y)$ is linear in both $x$ and $y$ individually, it is immediate that

$$
E f\left(\frac{S_{m}^{(X)}}{m}, \frac{S_{n}^{(Y)}}{n}\right)=f\left(E\left(\frac{S_{m}^{(X)}}{m}, \frac{S_{n}^{(Y)}}{n}\right)\right)=f(x, y)
$$

by also using the assumption that the $X_{i}$ 's are independent of the $Y_{j}$ 's. This then establishes the proof.

Theorem 2. If $f(t, s)$ is convex both in $t$ and $s$ in neighborhood of $(x, y)$, then when $m$ and $n$ are sufficiently large we have

$$
A_{m, n}(x, y)-\frac{C}{2}\left[\left(\operatorname{Var}\left(\frac{S_{m}^{(X)}}{m}\right)\right)^{\frac{1}{2}}+\left(\operatorname{Var}\left(\frac{S_{n}^{(Y)}}{n}\right)\right)^{\frac{1}{2}}\right]^{2} \leq f(x, y) \leq A_{m, n}(x, y)
$$

where $A_{m, n}(x, y)=E f\left(\frac{S_{m}^{(X)}}{m}, \frac{S_{n}^{(Y)}}{n}\right)$ is the approximation form and $C$ is a uniform bound of the second derivatives of $f(t, s)$ as given for Formula (3.2).

Proof. According to Formula (3.2), the left inequality holds. Therefore, proof only for $f(x, y) \leq A_{m, n}(x, y)$ is required. When $m$ and $n$ are sufficiently large, $\frac{S_{m}^{(X)}}{m}$ and $\frac{S_{n}^{(Y)}}{n}$ approach $x$ and $y$ respectively, and both have the joint distributional support in a neighborhood of $(x, y)$ wherein $f$ is convex in both components.

By iteratively using the well-known Jensen's inequality with the assumption that the $X_{i}$ 's are independent of the $Y_{j}$ 's, we have

$$
\begin{aligned}
E f\left(\frac{S_{m}^{(X)}}{m}, \frac{S_{n}^{(Y)}}{n}\right) & =E_{Y}\left[E_{X} f\left(\frac{S_{m}^{(X)}}{m}, \frac{S_{n}^{(Y)}}{n}\right)\right] \\
& \geq E_{Y}\left[f\left(E\left(\frac{S_{m}^{(X)}}{m}, \frac{S_{n}^{(Y)}}{n}\right)\right)\right] \\
& \geq f\left(x, E_{Y}\left(\frac{S_{n}^{(Y)}}{n}\right)\right)=f(x, y)
\end{aligned}
$$


which proves the theorem.

In practical applications of the bivariate approximation forms, there normally exist some known values of $f\left(x_{i}, y_{i}\right)$ at $\left(x_{i}, y_{i}\right), 1 \leq i \leq N$. Such values of $f\left(x_{i}, y_{i}\right)$ are the basis for obtaining an approximate of any $f(x, y)$. It is advisable that we apply the integral approximating forms, including the bivariate normal form and the bivariate gamma form, for doing the approximation task in view of the ease such two forms may offer. Let there be a bounded convex set $\Omega$ in $\mathbf{R}^{2}$ such that $\left(x_{i}, y_{i}\right), 1 \leq i \leq N$ are interior points. This convex set is normally obtainable. Then we may undertake a partition on $\Omega$ such that

$$
\Omega=\bigcup_{i=1}^{N} D_{i}
$$

where $D_{i}$ 's are disjoint and $\left(x_{i}, y_{i}\right)$ is an interior point of $D_{i}$.

For notational ease, let $\underset{\sim}{z}=(t, s)$ and $d \underset{\sim}{z}=d t d s$ to be the 2-dimensional differential. Then in light of Formula (2.5) we consider

$$
U_{m, n, N}(x, y)=\frac{\sqrt{m n}}{\sigma w} \sum_{k=1}^{N} f\left(x_{k}, y_{k}\right) \int_{D_{k}} \phi\left(\frac{\sqrt{m}(t-x)}{\sigma}\right) \phi\left(\frac{\sqrt{n}(s-y)}{w}\right) d \underset{\sim}{z}
$$

where $\phi$ is the probability density of standard normal distribution. Corresponding to Formula (2.6), we may similarly consider

$$
V_{m, n, N}(x, y)=\frac{m^{m} x^{-m} n^{n} y^{-n}}{(m-1) !(n-1) !} \sum_{k=1}^{N} f\left(x_{k}, y_{k}\right) \int_{D_{k}} t^{m-1} s^{n-1} e^{-\left(\frac{m t}{x}+\frac{n s}{y}\right)} d \underset{\sim}{z}
$$

while it should be noted that $\Omega$ should only be in the first quadrant of $\mathbf{R}^{2}$, where bivariate gamma form is defined.

Then $U_{m, n, N}(x, y)$ and $V_{m, n, N}(x, y)$ are the proposed approximates for $f(x, y)$ based on the known values of $f\left(x_{k}, y_{k}\right), 1 \leq k \leq N$.

Theorem 3. Regarding the approximates $U_{m, n, N}(x, y)$ and $V_{m, n, N}(x, y)$, we have

$$
\lim _{(m, n) \rightarrow(\infty, \infty)} U_{m, n, N}\left(x_{k}, y_{k}\right)=f\left(x_{k}, y_{k}\right)=\lim _{(m, n) \rightarrow(\infty, \infty)} V_{m, n, N}\left(x_{k}, y_{k}\right)
$$

for any $\left(x_{k}, y_{k}\right), 1 \leq k \leq N$.

Proof. Proof for $V_{m, n, N}$ can be established in similar way as the following for proving the case of $U_{m, n, N}$. According to the definition in above,

$$
\begin{aligned}
U_{m, n, N}\left(x_{j}, y_{j}\right) & =\sum_{k=1}^{N} f\left(x_{k}, y_{k}\right) \frac{\sqrt{m n}}{\sigma w} \int_{D_{k}} \phi\left(\frac{\sqrt{m}\left(t-x_{j}\right)}{\sigma}\right) \phi\left(\frac{\sqrt{n}\left(s-y_{j}\right)}{w}\right) d \underset{\sim}{z} \\
& =\sum_{k=1}^{N} f\left(x_{k}, y_{k}\right) \int_{E_{m, n, j, k}} \phi\left(t^{\prime}\right) \phi\left(s^{\prime}\right) d z_{\sim}^{\prime}
\end{aligned}
$$


where $E_{m, n, z, k}$ is the image of transforming $D_{k}$ by $t^{\prime}=\frac{\sqrt{m}\left(t-x_{j}\right)}{\sigma}$ and $s^{\prime}=\frac{\sqrt{n}\left(s-y_{j}\right)}{w}$ for varying $k$, while $j$ is fixed. When $k \neq j, 1 \leq k \leq N$, we see that as $(m, n) \rightarrow(\infty, \infty)$ all the element $\left(t^{\prime}, s^{\prime}\right)$ in $E_{m, n, j, k}$ falls at a distance of approaching infinity from the origin. This means that $\int_{E_{m, n, j, k}} \phi\left(t^{\prime}\right) \phi\left(s^{\prime}\right) d z_{\sim}^{\prime}$ has the probability value for an area at the extreme outskirt of standard bivariate normal distribution with zero correlation. Therefore, when $k \neq j$ we have

$$
\int_{E_{m, n, j, k}} \phi\left(t^{\prime}\right) \phi\left(s^{\prime}\right) d \underset{\sim}{z^{\prime}} \rightarrow 0 \text { as }(m, n) \rightarrow(\infty, \infty)
$$

On the other hand, when $k=j$ we can see that as $(m, n) \rightarrow(\infty, \infty) E_{m, n, j, k}$ extend out on both components to the full support of standard bivariate normal distribution with zero correlation. Therefore, when $k=j$ we have

$$
\int_{E_{m, n, j, k}} \phi\left(t^{\prime}\right) \phi\left(s^{\prime}\right) d \underset{\sim}{z} \rightarrow 1 \text { as }(m, n) \rightarrow(\infty, \infty)
$$

By summarizing the above results, we then have

$$
\begin{aligned}
\lim _{(m, n) \rightarrow(\infty, \infty)} U_{m, n, N}\left(x_{j}, y_{j}\right) & =\sum_{k=1}^{N} f\left(x_{k}, y_{k}\right) \lim _{(m, n) \rightarrow(\infty, \infty)} \int_{E_{m, n j, k}} \phi\left(t^{\prime}\right) \phi\left(s^{\prime}\right) d z_{\sim}^{\prime} \\
& =f\left(x_{j}, y_{j}\right) \cdot 1=f\left(x_{j}, y_{j}\right)
\end{aligned}
$$

which completes the proof for $U_{m, n, N}$.

It is apparent that Theorem 3 gives the assurance of consistency that the approximates of $f(x, y)$ at $(x, y)=\left(x_{k}, y_{k}\right)(1 \leq k \leq N)$ obtained by using the bivariate normal form or bivariate gamma form shall equal the originally given $f\left(x_{k}, y_{k}\right)$ 's when we let $(m, n) \rightarrow(\infty, \infty)$

\section{Some Example with Error Bounds}

For the purpose of actually feeling about the performance of the proposed bivariate approximation forms, we selectively show heretofore a few examples with error bounds provided.

(1) Example 1: $f(x, y)=x y, x \geq 0$ and $y \geq 0$.

We take bivariate Bernstein form. Then it gives

$$
\begin{aligned}
A_{m, n}(x, y) & =\sum_{\substack{0 \leq i \leq m \\
0 \leq j \leq n}} \frac{i t}{m} \frac{j s}{n}\left(\begin{array}{c}
m \\
i
\end{array}\right)\left(\begin{array}{c}
n \\
j
\end{array}\right)\left(\frac{x}{t}\right)^{i}\left(1-\frac{x}{t}\right)^{m-i}\left(1-\frac{y}{s}\right)^{j}\left(1-\frac{y}{s}\right)^{n-j} \\
& =\frac{t s}{m n} \cdot \frac{m x}{t} \cdot \frac{n y}{s}=x y=f(x, y)
\end{aligned}
$$

which is in line with the assertion of Theorem 1. 
(2) Example 2: $f(x, y)=e^{-(x+y)}$ for $x \geq 0, y \geq 0$.

The second derivatives are all bounded by $C=1$ everywhere in the defined domain. We take bivariate Poisson form. Then, by the fact that $f$ is convex in both $x$ and $y$, Theorem 2 implies

$$
\begin{aligned}
& \sum_{0 \leq i, j<\infty} e^{-\left(\frac{i}{m}+\frac{j}{n}\right)} \frac{(m x)^{i}(n y)^{j}}{i ! j !} e^{-(m x+n y)}-\frac{1}{2}\left(\frac{x}{m}+\frac{y}{n}+2 \sqrt{\frac{x y}{m n}}\right) \\
\leq & e^{-(x+y)} \leq \sum_{0 \leq i, j<\infty} e^{-\left(\frac{i}{m}+\frac{j}{n}\right)} \frac{(m x)^{i}(n y)^{j}}{i ! j !} e^{-(m x+n y)}
\end{aligned}
$$

(3) Example 3: $f(x, y)=\frac{1}{(1+x)(1+y)}$ for $x \geq 0$ and $y \geq 0$.

It is obvious that the second derivatives are all bounded by $C=2$ on the defined domain, and that $f(x, y)$ is convex in both $x$ and $y$. Therefore by taking bivariate normal form and by Theorem 2 , we have

$$
\begin{aligned}
& \frac{\sqrt{m n}}{2 \pi \sigma w} \int_{-\infty}^{\infty} \int_{-\infty}^{\infty} \frac{1}{(1+t)(1+s)} e^{-\frac{1}{2}\left[\frac{m(t-x)^{2}}{\sigma^{2}}+\frac{n(s-y)^{2}}{w^{2}}\right]} d t d s-\left(\frac{\sigma^{2}}{m}+\frac{w^{2}}{n}+2 \frac{\sigma w}{\sqrt{m n}}\right) \\
\leq & \frac{1}{(1+x)(1+y)} \leq \frac{\sqrt{m n}}{2 \pi \sigma w} \int_{-\infty}^{\infty} \int_{-\infty}^{\infty} \frac{1}{(1+t)(1+s)} e^{-\frac{1}{2}\left[\frac{m(t-x)^{2}}{\sigma^{2}}+\frac{n(s-y)^{2}}{w^{2}}\right]} d t d s
\end{aligned}
$$

\section{References}

[1] Abel, U. and Ivan, M., Asymptotic expansion of the multivariate Bernstein polynomials on a simplex, Approx. Theory Appl. (N. S.) 16(2000), 85-93.

[2] Farouki, R., Convergent inversion approximations for polynomials in Bernstein form, Computer Aided Geom. Design 17(1999), 179-196.

[3] Kao, C-S., Multivariate approximation forms extending Bernstein polynomials and beyond, (2002). (submitted)

[4] Ghosal, S., Convergence rates for density estimation with Bernstein polynomials, Ann. Statist. 29(2001), 1264-1280.

[5] Impens, C. and Vernaeve, H., Asyptotics of differentiated Bernstein polynomials, Constr. Approx. 17(2001), 47-57.

[6] Petrone, S., Bayesian density estimation using Bernstein polynomials, Canad. J. statist. 27(1999), 105-126.

[7] Walz, G., Asymptotic expansions for multivariate polynomial approximation, J. of Comput and Appl. Math. 122(2000), Issue:1-2, 317-328.

Department of Mathematics and Institute of Statistical Science National Chung Cheng University Minhsiung, Chiayi, Taiwan.

E-mail: scma@mthmp.math.ccu.edu.tw 